\title{
Papers
}

\section{Differences in p53 and Bcl-2 expression in relation to cell proliferation during the development of human embryos}

V Lichnovský, Z Kolář, P Murray, A Hlobilková, D Černochová, E Pospíšilová, B Vojtěšek, R Nenutil

Institute of Histology and Embryology,

Faculty of Medicine, Palacký University, Olomouc, Hnevotínská 3, CZ-77515, Czech Republic

V Lichnovský

D Černochová

E Pospíšilová

Institute of Pathology, Faculty of Medicine, Palacký University, Olomouc and Laboratory of Molecular Pathology, Centre of Molecular Biology and Medicine (CMBM), Palacký University, Olomouc, CZ-77515, Czech Republic

Z Kolár

A Hlobilková

School of Health

Sciences, University of Wolverhampton, Wolverhampton

WV1 1DJ, UK

P Murray

Laboratory of Molecular Biology, CMBM, Palacký University, Olomouc and Department of

Molecular and Cellular

Oncology, Masaryk

Memorial Cancer

Institute, Brno,

CZ-65653, Czech

Republic

B Vojtěšek

Women's Hospital, Department of Pathology, Brno, CZ-65617, Czech

Republic

R Nenutil

Correspondence to: Professor Lichnovský. e-mail: lichno@risc.upol.cz

Accepted for publication 26 March 1998

\begin{abstract}
Aims-To study the patterns of p53 and Bcl-2 expression in relation to cell proliferation during human embryogenesis in order to help elucidate their potential roles in the regulation of cell proliferation and apoptosis during morphogenesis. Methods-Immunohistochemistry for p53, Bcl-2, and proliferating cell nuclear cell antigen (PCNA) proteins was performed, using a variety of monoclonal antibodies, on paraffin wax embedded sections of tissues from 68 human embryos and fetuses of between 4 and 30 weeks gestation.

Results-Positive relations between sites of proliferative activity (as detected by PCNA expression) and p53 expression were found in the kidney, early developmental stages of intestine and lungs, liver, pancreas, heart, and in embryonic osteoblasts. On the other hand, positive relations between proliferative activity and Bcl-2 expression were found in the gonads, adrenal glands, in the cells of the dental lamina, hair follicles, syncytiotrophoblast, chondrocytes, and more advanced stages of intestinal development. In tissues of the central nervous system, p53 and Bcl-2 were co-expressed at the same sites but there was an inverse relation between p53/ Bcl-2 expression and proliferative activity. Conclusions-These data suggest that p53 and Bcl-2 have tissue specific and stage specific functions during embryogenesis. (f Clin Pathol: Mol Pathol 1998;51:131-137)
\end{abstract}

Keywords: immunohistochemistry; human embryogenesis; $\mathrm{p} 53$; Bcl-2

The p53 gene encodes a $53 \mathrm{kDa}$ nuclear phosphoprotein that is a negative regulator of the G1-S phase transition in the cell cycle. Tumour suppression by $\mathrm{p} 53$ is linked to a cell cycle check point induced by DNA damage, ${ }^{1}$ in which p53 can induce either growth arrest ${ }^{2}$ or apoptosis $^{3}$ in the damaged cells. The most important biochemical function of wild-type p53 is the sequence specific transactivation of target genes. ${ }^{4}$ The ability of p53 to activate transcription has led to the hypothesis that genes induced by p53 might mediate its biological role as a tumour suppressor. ${ }^{56}$ p53 mRNA has been demonstrated in a number of developing murine tissues ${ }^{78}$ and p53 protein concentrations have been shown to decrease as normal murine development progresses. ${ }^{9}$ p5 3 expression has also been demonstrated in young mice in tetraploid primary spermatocytes during the pachytene stages of meiosis. ${ }^{10}$ Donehower and colleagues ${ }^{11}$ showed that mice lacking a functional p53 gene develop normally but have a greater tendency to develop tumours of varying types although, more recently, p53 deficiency has been shown to be associated with a high frequency of developmental abnormalities in mice. ${ }^{12}{ }^{13}$ It has also been shown that accumulation of $\mathrm{p} 53$ protein occurs in early development in both irradiated and normal murine tissues. ${ }^{14}$ Thus, there are conflicting reports on the potential importance of p53 in development. In addition, there is little information available on the expression of p53 during the development of human embryos. ${ }^{15} 16$

Bcl-2, an integral membrane protein of the mitochondrial, nuclear, and endoplasmic reticular membranes, plays a crucial role in preventing apoptosis and in maintaining cell

Table 1 Details of embryos and fetuses examined

\begin{tabular}{|c|c|c|c|c|}
\hline \multirow[b]{2}{*}{ Age (weeks) } & \multirow{2}{*}{$\begin{array}{l}\text { Number of } \\
\text { samples }\end{array}$} & \multicolumn{3}{|c|}{$\begin{array}{l}\text { Immunohistochemical tests } \\
\text { performed }\end{array}$} \\
\hline & & PCNA & $p 53$ & Bcl-2 \\
\hline 4 & 1 & 1 & 1 & 1 \\
\hline 5 & 3 & 3 & 3 & 2 \\
\hline 6 & 11 & 7 & 8 & 4 \\
\hline 7 & 14 & 11 & 12 & 6 \\
\hline 8 & 7 & 7 & 7 & 6 \\
\hline 9 & 4 & 3 & 4 & 1 \\
\hline 10 & 4 & 3 & 3 & 4 \\
\hline 11 & 6 & 6 & 6 & 6 \\
\hline 12 & 4 & 4 & 4 & 1 \\
\hline 13 & 3 & 3 & 3 & 2 \\
\hline 14 & 1 & - & 1 & - \\
\hline 15 & 1 & 1 & 1 & - \\
\hline 16 & 1 & 1 & 1 & 1 \\
\hline 18 & 2 & 2 & 2 & 2 \\
\hline 25 & 3 & 3 & 2 & 3 \\
\hline 28 & 2 & 2 & 2 & 2 \\
\hline 30 & 1 & 1 & 1 & 1 \\
\hline Total $4-8$ & 36 & 19 & 31 & 19 \\
\hline Total 9-30 & 32 & 29 & 30 & 23 \\
\hline Total 4-30 & 68 & 58 & 61 & 42 \\
\hline
\end{tabular}


survival. ${ }^{17-19}$ The Bcl-2 mediated block of apoptosis might be the result of its influence on the release of cytochrome $\mathrm{c}$ from mitochondria. ${ }^{18}{ }^{19}$ Novack and Korsmeyer ${ }^{20}$ found Bcl-2 expression in a variety of murine embryonic tissues, such as cells of the bronchial stroma, intestinal crypt epithelium, urethral buds, and metanephrogenic caps. Expression of Bcl-2 has also been demonstrated in the retinal neuroepithelium, neuroblasts of embryonic brain and spinal cord, and in digital zone cells of the limb bud blasteme, but not in cells of the interdigital zone, where rapid cell death occurs during development. From the example of differentiating limbs, it is clear that apoptosis as well as Bcl-2 expression are involved in the morphogenesis of a number of organs. To date, no literature has been found on Bcl-2 expression in early stages of the human embryo (up to 12 weeks of maturation). One study ${ }^{21}$ described Bcl-2 expression in the organs of three older fetuses, but at this stage, morphological development is essentially complete.

Because both proteins are important regulators of cell growth and apoptosis, ${ }^{17}{ }^{21} 22$ a study of p53 and Bcl-2 expression in relation to proliferative rates (as measured by proliferating cell nuclear antigen (PCNA) expression), might provide data on their importance during the embryological development of various tissues and organs in humans.

\section{Methods}

Sixty eight human embryos and fetuses, all from normal pregnancies, of between 4 and 30 weeks gestation, were available as formal/saline or methacarn fixed paraffin wax embedded tissue blocks (table 1$)$. Sections $(6 \mu \mathrm{m})$ were prepared from each tissue block for immunohistochemistry. In embryos of advanced stages ( $>15$ weeks) all organs were removed and dissected before tissue fixation. Appropriate tissue preservation was confirmed by haematoxylin and eosin staining of all samples before immunohistochemistry. Any macerated or poorly preserved material was discarded.

Immunoperoxidase assays were performed on dewaxed sections using monoclonal antibodies PC-10, DO-1, DO-7, Bp53-12, and 124. Monoclonal antibodies DO-1, DO-7, and Bp53-12 2324 are specific for human p53, monoclonal antibody 124 (Dako Ltd, Glostrup, Denmark) is specific for Bcl-2, and monoclonal antibody PC10 (Dako Ltd) is used for the detection of PCNA.

Primary antibodies were detected using a sensitive streptavidin-biotin peroxidase system (Vectastain Elite kit; Vector Laboratories, Burlingame, California, USA) as recommended by the manufacturer. 3,3'Diaminobenzidine was used as the chromogen. Primary antibody was ommitted from negative controls. Positive controls for p53, PCNA, and Bcl-2 consisted of cases of breast cancer shown previously to express these markers.

The intensity of staining was noted and the numbers of positive cells were evaluated semiquantitatively by two independent investigators and classified as either negative (no cells stained), weakly stained $(<5 \%$ of cells posi- tive), moderately stained (between $5 \%$ and $30 \%$ of cells positive), or strongly stained ( $>30 \%$ of cells positive). The ratio of marker positive embryos to total embryo count was also determined.

\section{Results}

KIDNEY

Mesonephros (primitive kidney, embryo aged 4-8 weeks)

p53 was expressed in the nuclei of secretory canal epithelium and focally in the cells of primitive renal glomeruli. PCNA was also found at the same sites. The staining intensity of both markers dropped sharply at about 7 weeks, coinciding with the involution of the mesonephros. Bcl-2 expression was weak and heterogeneous throughout the mesonephros.

\section{Metanephros (mature kidney, fetal age 9-20}

weeks)

p53 expression (fig 1A) was focal in the nuclei of cells of the blasteme of the neogenic zone and in the cells of maturing proximal tubules, stronger in the glomerular podocytes and mesangial cells, but only occasional in cells of primitive collecting tubules differentiating as ureteric buds. PCNA was particularly prominent in the metanephrogenic blasteme (fig 2A) of the neogenic zone (in cells unfolding as nephrons) and was also present in cells differentiating as the final ducts or collecting tubules. Bcl-2 was expressed in the cytoplasm of cells of the metanephrogenic blasteme differentiating as the nephron (fig 1B).

\section{DIGESTIVE TRACT}

Primitive intestine

p53 was expressed focally in both epithelial and mesenchymal cells but only in a few of the embryos examined (fig 2C). After 10 weeks, expression of p53 decreased. PCNA was also expressed in epithelial and mesenchymal cells (fig 2B) from 4 weeks (the youngest embryos examined), and in the fetal period was mainly localised in basal enterocytes of Lieberkühn's crypts. Bcl-2 was found in the cells of the primitive surrounding mesenchyma (fig 1C) in basal enterocytes of villi (fig 1D) and differentiating crypts of Lieberkühn during the fetal period (9-20 weeks).

LIVER

p53 was seen in some hepatocytes (fig 1E) of earlier developmental stages. In more advanced embryos, p53 expression decreased sharply and after 12 weeks of intrauterine life was seen only in isolated hepatocytes in the periphery of liver parenchyme. Some p53 expression was found in liver blood islets. PCNA was expressed weakly in hepatocytes during earlier phases (4-6 weeks), but in later periods was only expressed focally in the periphery of liver parenchyme, in cells of blood islets, and in mesenchymal cells of differentiating portal canals (fig $1 \mathrm{~F}$ ). Bcl-2 was found only in a few mesenchymal cells of portal canals. 

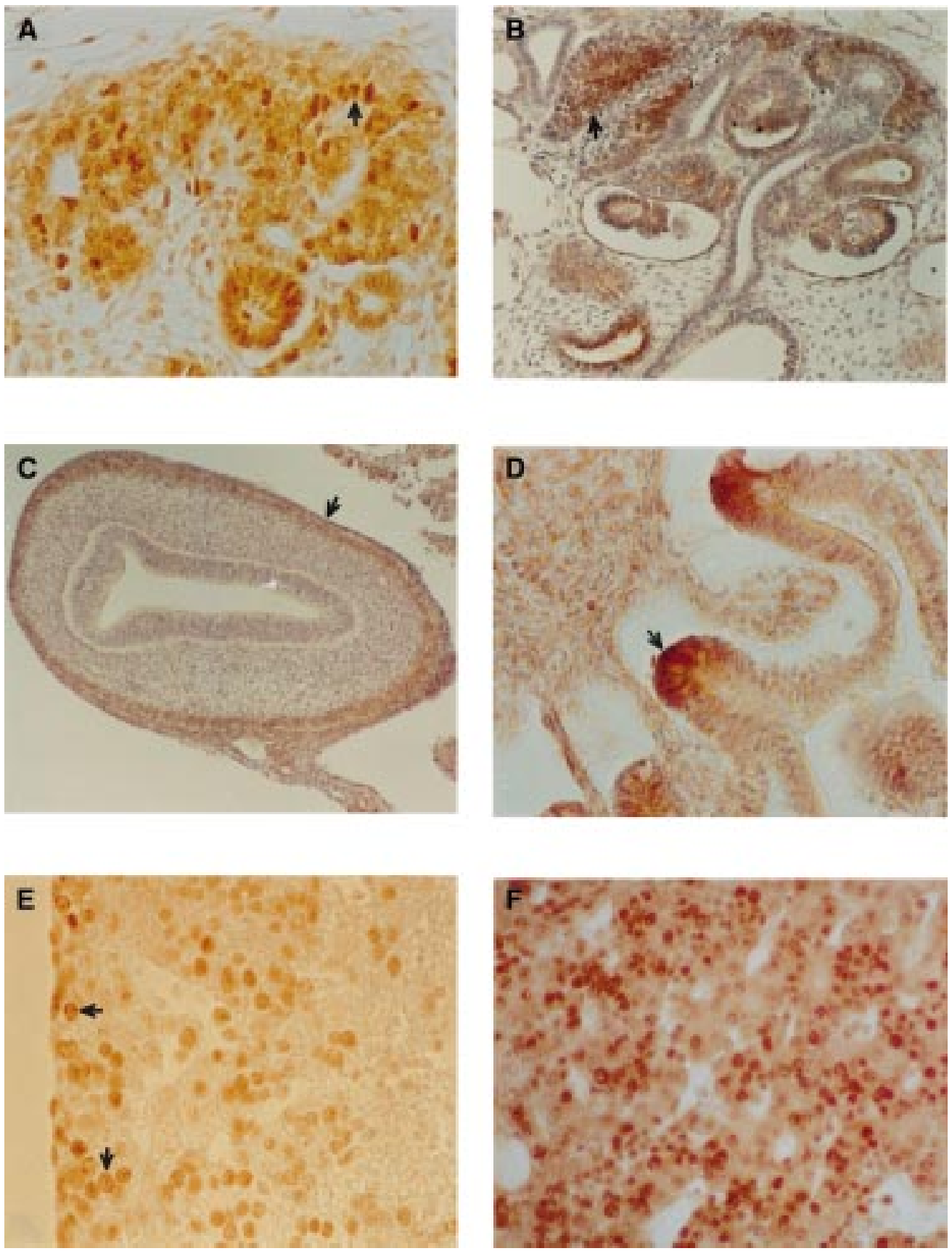

Figure 1 (A) Expression of $p 53$ (arrow) in the "neogene zone" of a human fetal kidney (11 week old fetus). (B) Expression of Bcl-2 in a human fetal kidney. Distinct expression is visible in the cells (arrow) of the metanephrogenic blasteme (12 week old fetus). (C) Expression of Bcl-2 in mesenchymal cells (arrow) of the anlage of the embryonal intestine (5 week old embryo). (D) Bcl-2 expression in the cells of differentiating crypts of Lieberkïhn (arrow) and in the cells of primitive intramural ganglia (18 week old fetus). (E) Expression of p53 (arrow) in outer zone of fetal liver. The intensity of the expression decreases quite distinctly from the superficial to the deeper layer (9 week old fetus). (F) PCNA positive nuclei of hepatocytes in the primitive liver cords (12 week old fetus).

PANCREAS

p53 was demonstrated in cells of differentiating glandular epithelium, surrounding primitive mesenchyme, and the vessel wall. The intensity of expression dropped sharply during development. PCNA was found in the same localisation but the intensity of staining was much greater and did not change during the course of embryogenesis. Weak expression of $\mathrm{Bcl}-2$ was seen in cells of the terminal part of the differentiating glandular epithelium and in the primitive mesenchyme. More intense expression was detected at earlier stages.
Thus, in liver and pancreas, $\mathrm{p} 53$ expression was found preferentially in sites with high proliferative activity, particularly during the earlier developmental stages. The locations for Bcl-2 expression did not correspond precisely with those of p53 but did match the expression of PCNA in more advanced fetal intestine.

LUNG

p53 was found in cells of primitive bronchial epithelium and surrounding mesenchyme with a dramatic decline during development, particularly after 12 weeks of intrauterine life. 

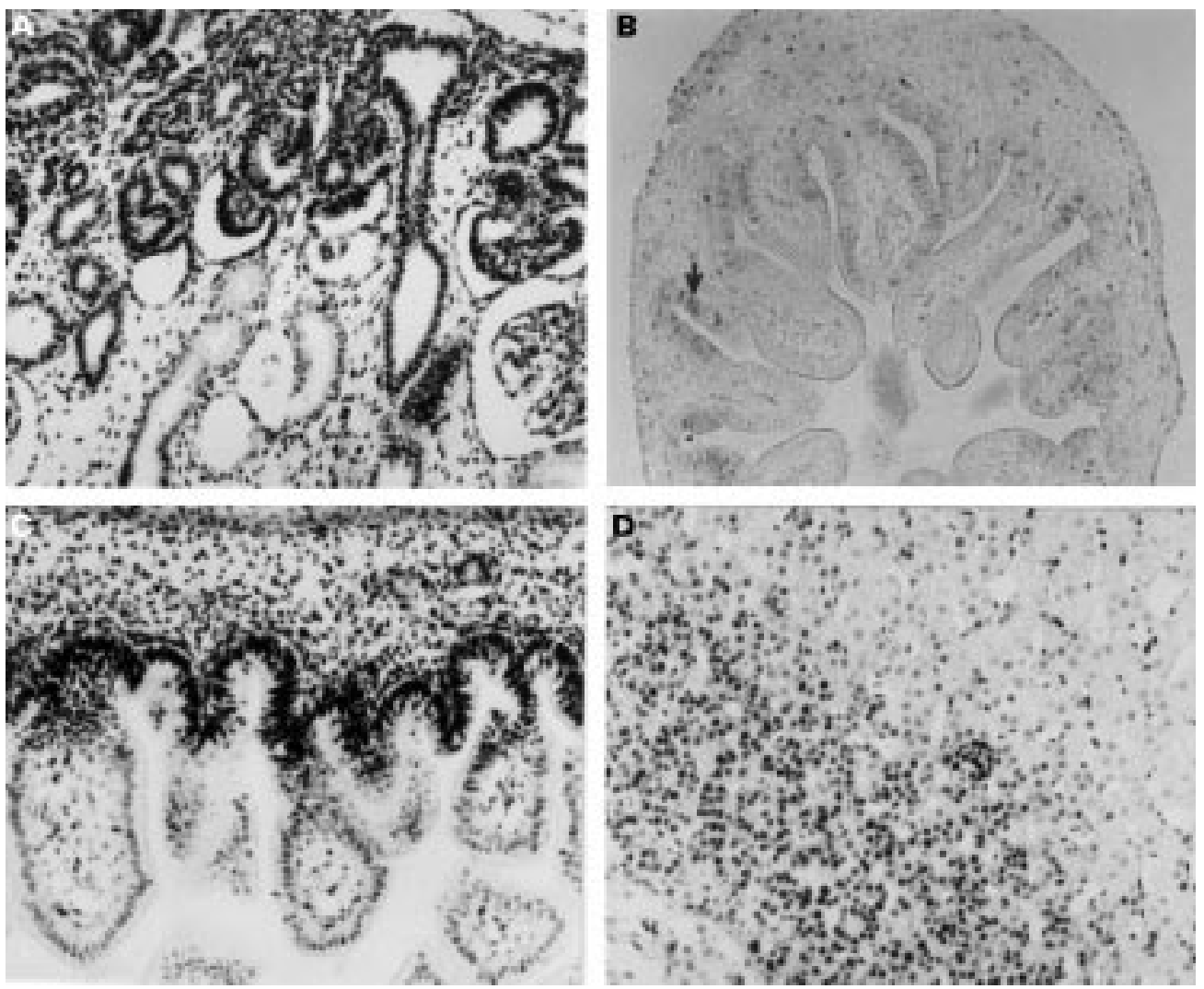

Figure 2 (A) Expression of PCNA in the "neogene zone" of human fetal kidney (10 week old fetus). (B) Expression of PCNA in the mesenchymal and epithelial cells of the fetal intestine (13 week old fetus). (C) p53 expression of scattered cells (arrow) of embryonal intestine (7 week old embryo). (D) Expression of PCNA in the superficial zone of the primitive cortex of the fetal adrenal gland (11 week old fetus). Thus, in the mesonephros, the topography of p53 expression corresponded closely with PCNA expression but not with Bcl-2 expression and the expression of both proteins decreased during development. In the metanephros, the expression of all three markers appeared in the same topographical regions without distinct changes during development.

PCNA was expressed in the same regions and also weakly in pulmonary blood vessel walls. The intensity of expression did not vary with developmental stage. Bcl-2 showed only weak expression in the mesenchymal cells surrounding the bronchial stem (fig $3 \mathrm{~A}$ ).

ADRENAL GLAND

p53 expression was not found. PCNA was conspicuous in cells of the superficial zone of the primitive cortex (fig 2D). In deeper parts of the cortex, PCNA expression was less evident. From 10 weeks of intrauterine life, it was also present in foci of the medullary cells and primitive sinusoids. Bcl-2 expression was seen only in foci of medullary cells (fig 3B).

GONADS

p53 was expressed very weakly in coelomic and mesenchymal cells of the primitive gonad and expression disappeared upon maturation. PCNA was expressed very strongly in the gonocytes and coelomic cells of medullary bands found in early stages ( $4-8$ weeks) but decreased during development. Bcl-2 colocalised with PCNA.

HEART

The $\mathrm{p} 53$ protein was demonstrated in primitive myoblasts of both compact and spongy layers but concentrations diminished as development progressed. It was also found sporadically in epicardial and endocardial cells. PCNA was noted in the same localisation but with an intensity gradient decreasing from the spongy to compact layer. Bcl-2 expression was not seen.

PRIMITIVE ORGANS OF THE CENTRAL NERVOUS SYSTEM (CNS)

p53 was found during the earlier stages of development (4-8 weeks) in the cells of the lamina epithelialis, weakly in the mesenchyme of the choroid plexus, in isolated cells of the cerebral cortex, in cells of the bulbus olfactorius, in the spinal ganglia (figure $3 \mathrm{C}$ ), in the mantle layer of the spinal cord, and in ectodermal cells of otocysts. In more advanced fetuses (9-30 weeks), p53 expression disappeared dramatically, with the exception of only one case in which expression was weak. PCNA expression was found in the cells of the germinal zone of the CNS anlage organ during embryogenesis and was also marked in fetal nervous tissues, mostly in the primitive cerebellar cortex, parahippocampal gyrus, thalamus, choroid plexus, cerebral cortex, and brain stem. Bcl-2 was expressed in the neuroblasts of the germinal and mantle zones of the spinal cord (fig 3D), of the brain (fig 3E) and spinal ganglia, the cells of the choroid plexus, otocysts, and primitive retina. These showed a tendency towards a stage dependent decrease. Thus, in the CNS, an inverse relation was seen between sites of 

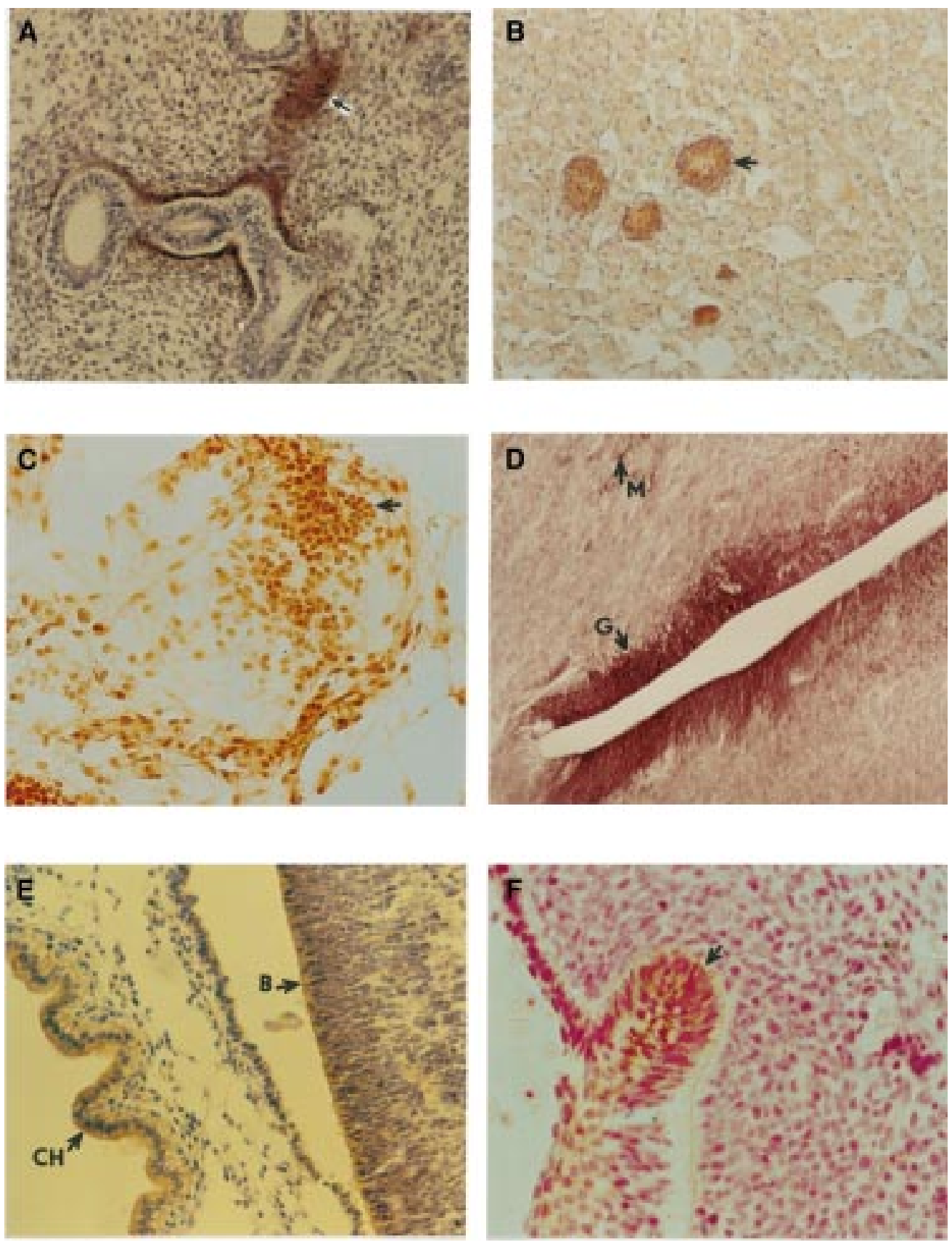

Figure 3 (A) Expression of Bcl-2 (arrow) in the mesenchymal cells surrounding the bronchial stem (11 week old fetus). (B) Expression of Bcl-2 in the cells (arrow) of the medular anlage differentiating adrenal gland (11 week old fetus). (C) Expression of p53 in some cells (arrow) of a spinal ganglion anlage (6 week old embryo). (D) Expression of Bcl-2 in the cells of the germinal $(G)$ and mantel $(M)$ zone of an embryonal spinal cord (7 week old embryo). (E) Expression of Bcl-2 in the neuroblasts of brain cortex (B) and the cells of choroid plexus (CH) (8 week old embryo). (F) Bcl-2 expression of the cells of the epithelial bud (arrow) of the dental crest (6 week old embryo).

p53 and PCNA expression. During earlier stages of development, p53 and Bcl-2 were often co-expressed.

OTHER TISSUES AND ORGANS

p53 expression was found in the trabecular osteoblasts of the cranial skeleton in fetal developmental stages. PCNA expression corresponded closely with p53 and/or Bcl-2. Bcl-2 was expressed in cells of epithelial buds in the dental crest (fig 3F), ectodermal cells of the anlage of hair follicles, syncytiotrophoblast, and chondrocytes of the digital zone of limb anlages.

\section{Discussion}

We have shown that genes involved in the regulation of the cell cycle of human adults are active from the earliest phases of embryogenesis. The reason for the massive accumulation of the p53 product in embryonic cells, detectable using immunohistochemical methods, is not clear but might testify to its important role in morphogenesis. The finding of greater amounts of the p53 protein in early embryos (before the end of organogenesis) supports the idea that p53 controls the elimination of cells with genetic abnormalities 
during the early stage of embryogenesis, possibly by inducing p 53 mediated apoptosis. ${ }^{25}$

We found immunohistochemical staining of p53 in only a proportion of embryos examined. This might reflect the earlier finding that $\mathrm{p} 53$ is not absolutely necessary for development or regulation of the cell cycle in all embryos, ${ }^{11}$ and also that some familial differences in the ability to utilise or synthesise $\mathrm{p} 53$ could be involved. It is also possible that variability of tissue processing might have prevented the detection of low concentrations of $\mathrm{p} 53$ in some cases, and a more sensitive technique might prove successful here. ${ }^{14}$

It is well known that wild-type p53 has a very short half life. However, it may be stabilised during DNA repair ${ }^{14}$ induced by a variety of mutagenic agents, including gamma and UV irradiation. ${ }^{26}{ }^{27}$ Although it is not likely that extensive DNA damage could explain p53 accumulation in our samples, we cannot fully exclude the possibility that p53 might be induced by DNA damage or upregulated in response to fetal trauma. ${ }^{16}$

There are other known mechanisms responsible for stabilisation of $\mathrm{p} 53$, which are also associated with its inactivation. According to recent data, p53 inactivation is usually the result of deletion and mutation, and only occasionally caused by independent bi-allelic point mutations. ${ }^{28}$ Inactivation might be the result of alterations in other genes that influence p53 activity, such as mdm-2 oncogene overexpression and products of viral E6 and ElB genes. ${ }^{28}{ }^{29}$ However, these mechanisms are not likely to be operative during embryonic life. Macleod and colleagues ${ }^{30}$ have studied the p53 dependent cell death pathway in the developing mouse nervous system and have demonstrated the importance of the retinoblastoma $(\mathrm{Rb})$ gene on $\mathrm{p} 53$ protein induction. We can also speculate about synthesis of more stable embryonic isotypes of p53. Our results suggest that p53 expression in specific organs and tissues and at specific stages of development is probably important. The importance of p53 control in the development of specific tissues is underlined by recent studies showing that p53 overexpression causes defects in kidney development. ${ }^{31}$ In addition, Bcl-2 mediated regulatory pathways might be involved in protection from cell death at specific times during the development of some organs. Our results show that p53 and Bcl-2 were expressed simultaneously in human embryonic tissues only in the central nervous system, suggesting that both proteins are important regulators of CNS development. This is supported by recent data showing that CNS abnormalities are common in p53 null mice ${ }^{1213}$ and that neuronal differentiation is affected by $\mathrm{p} 53$ concentrations. ${ }^{32}$

In some developing tissues, p53 expression correlated positively with sites of high proliferative activity. In these tissues, higher p53 expression might be a reflection of regulatory pathways operating to subdue "inadequate" proliferation, or it may be necessary for the control of the higher rate of genomic mistakes that might arise more frequently at sites of intense cell division. It would be interesting to elucidate whether the rate of apoptosis is altered in these tissues. In a few components of the CNS there was an inverse relation between proliferation and p53 expression. Thus, in some development stages of the CNS, p53 might play a less important role and its regulatory effects might be substituted by other regulatory genes, including the Bcl-2 gene, which protects irreplaceable neural cells against death.

The high expression of both p53 and Bcl-2 in the presence of low level cell proliferation seen in some embryonic and fetal tissues might reflect attempts to ensure survival of the cells during critical periods of development. The evaluation of the localisation and quantity of Bcl-2 expression also demonstrated that only in some organs and cell types (more advanced intestine, gonads, dental crest, hair follicles, syncytiotrophoblast, and chondrocytes) did the number of Bcl-2 positive cells correlate positively with proliferative activity. We assume, therefore, that in these tissues Bcl-2 might ensure cell survival or participate in the control of cell division.

Our results support the hypothesis that embryogenesis is under multiple levels of control. ${ }^{14}$ This control could be mediated for each organ and developmental stage by different regulatory pathways that trigger time dependent promotion of proliferation, differentiation, and/or apoptosis. These findings might also have implications for the study of tissue susceptibility to teratogenic and carcinogenic insults.

Supported in part by grants 301/96/K047 and 312/96/0355 from Grant Agency of Czech Republic, VS 96154 from Agency for University Research Support, and 3477-3 Internal Grant Agency of Czech Ministry of Health.

1 Kastan MB, Onyekwere O, Sidransky D, et al. Participation of 553 protein in the cellular response to DNA damage. Cancer Res 1991;51:6304-11.

2 Clark AR, Purdie CA, Harrison DJ, et al. Thymocyte apoptosis induced by $\mathrm{p} 53$ dependent and independent pathways. Nature 1993;362:849-52.

3 Lowe SW, Schmitt EM, Smith SW, et al. p53 is required for radiation induced apoptosis in mouse thymocytes. Nature 1993;362:847-9.

4 Raycroft L, Wu H, Lozano G. Transcriptional activation by wild-type but not transforming mutants of the p53 anti-oncogene. Science 1990;249:1049-51.

5 Fields S, Jang SK. Presence of a potent transcription activating sequence in the p53 protein. Science 1990;249: 1046-9.

6 Vogelstein B, Kinzler KW. p53 function and dysfunction. Cell 1992;70:523-6.

7 Rogel A, Popliker M, Webb CG, et al. p53 cellular tumour antigen: analysis of mRNA levels in normal adult tissues, embryos and tumours. Mol Cell Biol 1985;5:2851-5.

8 Schmid P, Lorenz A, Hameister H, et al. Expression of p53 during mouse embryogenesis. Development 1991;113:85765.

9 Louis JM, McFarland VW, May P, et al. The phosphoprotein p53 is down regulated post-transcriptionally during embryogenesis in vertebrates. Biochem Biophys Acta 1988;950: 395-402.

10 Schwartz D, Goldfinger N, Rotter V. Expression of p53 protein in spermatogenesis is confined to the tetraploid pachytene primary spermatocytes. Oncogene 1993;8:148794.

11 Donehower LA, Harvey M, Slagle BL, et al. Mice deficient for p53 are developmentally normal but susceptible to spontaneous tumours. Nature 1992;356:215-21.

12 Armstrong J, Kaufman MH, Harrison DJ, et al. High frequency developmental abnormalities in p53 deficient mice. Curr Biol 1995;5:931-6.

13 Sah VP, Attardi LD, Mulligan GJ, et al. A subset of p53 deficient embryos exhibit exencephaly. Nat Genet 1995;10: $175-80$. 
14 MacCallum DE, Hupp TR, Midgley CA, et al. The p53 response to ionising response to ionising radiation in adult
murine tissues. Oncogene 1996;13:2575-8.

15 Tauber Z, Lichnovský V, Kolár Z, et al. PCNA and p53 in tissues and organs of human embryos. Anat Anz 1996; 178(suppl): 94

16 Hall PA, Lane DP. Tumour suppressors: a developing role for p53? Curr Biol 1997; 7:R144-7.

17 Hockenbery DM, Oltvai ZM, Yin X-M, et al. Bcl-2 functions in an anti-oxidant pathway to prevent apoptosis. Cell 1993;75:241-51.

18 Kluck RM, Bossy-Wetzel E, Green DR, et al. The release of cytochrome c from mitochondria: a primary site for Bcl-2 cytochrome c from mitochondria: a primary site

19 Yang J, Liu X, Bhalla K, et al. Prevention of apoptosis by Yang J, Liu X, Bhalla K, et al. Prevention of apoptosis by Bcience 1997;275:1129-36.

20 Novack DV, Korsmeyer SJ. Bcl-2 protein expression during murine development. Am $\mathcal{F}$ Pathol 1994;145:61-73.

21 LeBrun DP, Warnke RA, Cleary ML. Expression of Bcl-2 in fetal tissues suggests a role in morphogenesis. Am $\mathcal{F}$ Pathol 1993;142:743-53.

22 Bartek J, Bartkova J, Vojtesek B, et al. Aberrant expression of the p53 oncoprotein is a common feature of a wide the p53 oncoprotein is a common feature of a wide 703.

23 Vojtesek B, Bartek J, Midgley CA, et al. An immunochemical analysis of the human nuclear phosphoprotein p53. New monoclonal antibodies and epitope mapping using recombinant p53. F Immunol Methods 1992;151 237-44.
24 Momand J, Zambetti GP, Olson DC, et al. The mdm-2 oncogene product forms a complex with p53 and inhibits p53-mediated transactivation. Cell 1992;69:1237-45.

25 Norimura T, Nomoto S, Katsuki M, et al. p53-dependent apoptosis suppresses radiation-induced teratogenesis. Nat Med 1996;2:577-80.

26 Fritsche M, Haessler C, Brandner G. Induction of nuclear accumulation of the tumour suppressor protein p53 by DNA-damaging agents. Oncogene 1993;8:307-18.

27 Hall P, McKee PH, du Menage H, et al. High levels of p53 protein in UV-irradiated normal human skin. Oncogene 1993;8:203-7.

28 Davidoff AM, Humphrey PA, Iglehart JK, et al. Genetic basis for p 53 overexpression in human breast cancer. Proc Natl Acad Sci USA 1991;88:5006-10.

29 Crook T, Farthing A, Vousden K. HPV-16 and cervical intraepithelial neoplasia. Lancet 1992;339:1231.

30 Macleod KF, Hu Y, Jacks T. Loss of Rb activates both p53dependent and independent cell death pathways in the developing mouse nervous system. EMBO 7 1996;15: developin

31 Godley LA, Kopp JB, Eckhaus M, et al. Wild-type p53 transgenic mice exhibit altered differentiation of the ureteric bud and possess small kidney. Genes Dev 1996;10: 836-50.

32 Eizenberg O, Faber-Elman A, Gottlieb E, et al. p53 plays a regulatory role in differentiation and apoptosis of central nervous system-associated cells. Mol Cell Biol 1996;16: 5178-85.

\section{Fournal of Clinical Pathology - http://www.molpath.com}

Visitors to the world wide web can now access the fournal of Clinical Pathology either through the BMJ Publishing Group's home page (http://www.bmjpg.com) or directly by using its individual URL (http://www.molpath.com). There they will find the following:

- Current contents list for the journal

- Contents lists of previous issues

- Members of the editorial board

- Information for subscribers

- Instructions for authors

- Details of reprint services.

A hotlink gives access to:

- BMJ Publishing Group home page

- British Medical Association web site

- Online books catalogue

- BMJ Publishing Group books.

The web site is at a preliminary stage and there are plans to develop it into a more sophisticated site. Suggestions from visitors about features they would like to see are welcomed. They can be left via the opening page of the BMJ Publishing Group site or, alternatively, via the journal page, through "about this site". 